\title{
WELFARE OF FARM ANIMALS
}

\begin{abstract}
HE range of subjects dealt with by the Universities Federation for Animal Welfare, as shown by the Spring issue of the U.F.A.W. Courier, appears to be worth special comment in that some of them may be taken for granted by many scientific workers or given less than the necessary attention.

A reprint from Nature, September 30, 1961, about the symposium on the assessment of pain organized by the Federation, is apposite in a publication of its own and an article based on a lecture given by E. C. Straiton at its summer congress, 1961, on "Unnecessary Cruelties among Farm Animals", which gives emphasis to matters that are constantly under-emphasized by many people.

Straiton's theme is that farm people often, or more than often, assume too easily that the reactions of animals to pain are vastly less than might be guessed by comparison with those of human beings and that, as a corollary, there is little or no objection to the infliction of cruelty when it is done in the guise of attention to the welfare of the individual. This could be described as a cutting article, and, perhaps, this approach was suitable in order to give emphasis to many practices which are in constant operation,
\end{abstract}

many of them receiving some sort of sanction in that they have been accepted for a very long time. $\mathrm{He}$ stated that the vast majority of working farm dogs are eruelly treated, inadequately fed, kept tied up with no water available, very often on a short chain and inadequately sheltered. He gave many instances in his experience of stupid, unskilled use of great force in attempts to calve cows, brutality in castrating farm animals, and other practices. $\mathrm{He}$ gave strong personal opinions about discomforts and worse from housing cloven-footed animals on accommodation with slatted floors, about the use of instruments for castrating by crushing the tissues, and about the methods of raising calves for marketing as veal by the use of the new methods designed to provide economical farm practice and at the same time veal of the type that appeals to the retail purchaser.

The issue reports Sir Thomas Dalling's account, given at the summer congress, of the international bodies which are concerned with veterinary activities designed to improve the standards and economy of animal husbandry by attention to animal health in the under-developed countries. W. A. Poor

\section{AGGREGATION OF BLOOD PLATELETS BY ADENOSINE DIPHOSPHATE AND ITS REVERSAL}

\author{
By Prof. G. V. R. BORN
}

Department of Pharmacology, Royal College of Surgeons of England, London

IN 1956 it was shown that blood platelets contain adenosine triphosphate (ATP) in extraordinarily high concentrations ${ }^{1}$ and that most of this ATP breaks down rapidly when platelets are suspended in plasma which is clotting ${ }^{2,3}$ or whenever platelets undergo viscous metamorphosis ${ }^{4}$. The suggestion was made ${ }^{5}$ that if this breakdown involved ATP situated on the surface of the platelets, the large net negative charge on platelets ${ }^{6}$ might be diminished and their tendency towards adhering to each other enhanced.

Recently, it has been found that platelets can be made to aggregate in plasma and to adhere to other surfaces by adding adenosine diphosphate (ADP) (ref. 7). Twenty-two nucleotides were tested for this effect; it was brought about only by ADP and by deoxyadenosine diphosphate. Since it is likely that ADP is produced by platelets themselves whenever their ATP breaks down, the adhesion and aggregation of platelets that occur in the initial stages of thrombosis may well be initiated by ADP. This effect has therefore been investigated by a method, recently described $^{8}$, with which the rate at and extent to which platelets aggregate can be determined quantitatively.

About $80 \mathrm{ml}$. of human blood was mixed with just enough sodium citrate $(3.8 \mathrm{mgm} . / \mathrm{ml}$. blood) or hoparin $(0.01 \mathrm{mgm} . / \mathrm{ml}$. blood) to prevent clotting. As cooling increases the tendency of platelets to aggregate, the experiments were done at room temperature of $20^{\circ}-22^{\circ}$ C. The blood was centrifuged at $500 \mathrm{~g}$ for $20 \mathrm{~min}$. The plasma, usually about $35 \mathrm{ml}$. and containing $10^{8}-10^{9}$ platelets $/ \mathrm{ml}$, was transferred with a siliconed capillary pipette into a plastic measuring cylinder.

A sample of $3 \mathrm{ml}$. was pipetted into a Spinco centrifuge tube, made of transparent plastic, which was inserted into a Unicam $S P 400$ absorptiometer. Light at a wave-length of $600 \mathrm{~m} \mu$ was passed through the tube. The dark current was set at infinity and the optical density of distilled water at zero. The optical density of platelet-rich plasma was proportional to the concentration of platelets in it, provided the optical density of platelet-free plasma was subtracted.

The platelet-rich plasma was stirred by a small iron rod covered in polythene which was rotated magnetically. Stirring was stopped when readings were taken. When plasma was stirred gently there was no significant change in the concentration of platelets or in the optical density; this showed that the platelets did not stick to the tube or to the stirrer.

When platelet-rich plasma was stirred vigorously the concentration of platelets usually decreased slightly and so did the optical density (Fig. 1). The decrease was mostly in the first half-hour; after that it was very slow or absent. Presumably a small proportion of the platelets was broken up by vigorous stirring.

All additions were made to the plasma while it was being stirred. When substances were added which caused the platelets to aggregate, the rate and extent of aggregation increased as the rate of stirring was increased up to about 1,000 r.p.m. In the experiments described here stirring was always at this rate. 